\title{
Sir Norman Lockyer's Contributions to Astrophysics.
}

\author{
By Prof. A. Fowler, F.R.S.
}

BY the death of Sir Norman Lockyer the B science of astrophysics has lost the energising and stimulating influence of the last of the great pioneers whose labours opened the way to so vast an extension of our knowledge of the universe. The science of celestial chemistry and physics had its real beginning in 1859 , when Kirchhoff's famous experiment on the reversal of spectral lines furnished the key to the interpretation of the dark lines of the solar spectrum, and thence to the determination of the composition of the sun and stars. During the earlier years the outstanding features in the development of the new science were the brilliant investigations of Huggins on the spectra of stars and nebulæ, and those of Rutherfurd and Secchi on the spectroscopic classification of the stars. Curiously enough, the sun had received but little attention during this period, and Lockyer was practically entering a virgin field when, in 1866 , he attached a small spectroscope to the modest $6-i n$, equatorial of his private observatory, and observed the spectrum of a sun-spot independently of the rest of the solar surface. Simple as it may now seem, this process of "taking the sun to bits," as Sir Norman used to call it, was an advance of fundamental importance. It not only gave an immediate and decisive answer to the question as to the cause of the darkness of sun-spots which was then under vigorous discussion in England and France, but also very soon led to the famous discovery of the method of observing solar prominences without an eclipse, with which Lockyer's name, in conjunction with that of Janssen, will for ever be associated. The story of this epoch-making observation has been told too often to need repetition, but it should not be forgotten that the principle of the method had been clearly recognised by Lockyer two years before he succeeded in obtaining a spectroscope suitable for the purpose in view.

Those who have become familiar with the beautiful solar phenomena presented by this method of observation will best understand the enthusiasm and delight with which Lockyer continued his observations whenever the sun was visible. On the first day of observation-October 20, I868he had identified the $\mathrm{C}$ and $\mathrm{F}$ lines of hydrogen, and a yellow line near $\mathrm{D}$, in the spectra of the prominences, and on November 5 he discovered that the prominences were but local upheavals of an envelope entirely surrounding the photosphere, to which he gave the name of the chromosphere, as being the region in which most of the variously coloured effects are seen during total eclipses of the sun. The peculiarities of the bright $F$ line at once suggested to his fertile mind that the spectroscope might disclose the physical state, as well as the chemical composition of the chromoNO. 2652 , VOL. IO5] sphere and prominences, through the medium of laboratory experiments, and from this beginning the close association of the laboratory with the observatory became the dominant note in his life's work. His first experiments were made in collaboration with his friend Frankland, and it was shown that the widening of the $\mathrm{F}$ line at the base of the chromosphere was to be accounted for by an increase of pressure. These experiments further demonstrated that the yellow line of the chromosphere, which had been named $D_{3}$, was quite distinct from hydrogen, and the then unknown gas to which it was to be attributed was given the now well-known name of helium. Up to the year I873, however, Lockyer's work was carried on almost entirely in his private observatory, and in the laboratory which he had established in his house at Hampstead. He not only continued his solar observations with conspicuous success, but also commenced his well-known "Researches on Spectrum Analysis in Connection with the Spectrum of the Sun," in which he developed experimental methods which afterwards became common practice.

On his transfer to South Kensington, with which his connection continued for forty years, the facilities at Lockyer's disposal for research were at first very meagre, but additions to equipment and staff were made from time to time, and in the later years the observatories and laboratories were well adapted for their special purposes. Lockyer's dream of becoming director of a permanent astrophysical observatory, comparable with those established by Governments in other countries, however, was never realised, and his work throughout was carried on in temporary buildings, and for the greater part of the time with modest grants in aid from year to year. In I9I2, on the transfer of the Solar Physics Observatory to the control of the University of Cambridge, Lockyer, in spite of his weight of years, courageously set about the erection of a new observatory at Sidmouth, and continued his work on stellar spectra almost to the close of his life. It is a lamentable fact that much of his time and energy was almost continually taken up with a struggle to obtain adequate means to carry on his researches.

The contributions to astrophysics made by Lockyer during nearly sixty years of strenuous endeavour in its various fields of investigation form the subject-matter of more than 200 papers and memoirs, and it is only possible here to refer to some of the larger questions in which he was specially interested. His work, both in the laboratory and in the observatory, was largely guided by bold speculations, which he was usually careful to regard as working hypotheses, and from time to time the main points were brought together 
in appropriate sequence in the form of books, among which are "The Chemistry of the Sun" (1887), "The Meteoritic Hypothesis" (1890), and "Inorganic Evolution" (1900). His observations and his views on their significance were thus made widely known, and the trend of his work could be the more readily followed. It was especially his desire to impress upon chemists and physicists the importance of the sun and stars as a means of investigating the behaviour of matter at high temperatures, and as possibly throwing light upon the nature of atoms and molecules.

Among the researches which have had the most potent influence, and have led to very definite advances, were those which dealt with the changes in the spectrum of the same element under different conditions of experiment. Lockyer was early led by his solar observations to a comparative study of the flame, arc, and spark spectra of some of the metallic elements, and one of his first successes was to show that some of the lines most characteristic of solar prominences, other than those of hydrogen and helium, were produced only under high temperature conditions, while some of those prominently affected in sun-spots were produced at a low temperature. With these and other observations as a basis he put forward, in 1873, his well-known dissociation hypothesis, which became the subject of much discussion. The hypothesis supposed that at successively higher temperatures the "molecular groupings" which existed at lower stages were broken up into finer forms of matter, or possibly into new elements, producing different spectral lines, and on this view it was shown that a multitude of solar observations which had seemed to be wholly inexplicable on the ground of previous laboratory experience became easy of explanation. Thus his view of the construction of the solar atmosphere was that if we could observe a section of it we should see it divided into a number of layers, each with its appropriate spectrum, and the spectrum would be simpler the nearer the layer was to the photosphere. The metallic elements, instead of existing as such in a reversing layer, were considered to be entirely broken up in the vicinity of the photosphere, and their germs distributed throughout the atmosphere, the molecular groupings becoming more complex as they became further removed from the source of heat. The theory doubtless calls for some re-statement in the light of modern views as to the structure of atoms and the origin of spectra, but it was a valuable guide to observation, and Lockyer anticipated the conclusion reached by St. John in recent years, that the complete absorption of any one element in the solar spectrum is the integration of lines special to various levels in the solar atmosphere. Lockyer himself seems to have been convinced that the ultimate products of dissociation were hydrogen and helium; but although this is so closely in accord with recent work on the structure of atomic nuclei, it does not seem probable that the phenomena studied by Lockyer were directly NO. 2652 , VOL. IO5] related to those investigated by Rutherford. The writer well remembers numerous attempts to produce the lines of hydrogen or of helium by the passage of powerful condensed discharges between metallic electrodes, all of which, however, were unsuccessful.

Work on the varying spectra of the elements was vigorously resumed by Lockyer in connection with the interpretation of the photographs of the chromospheric spectrum which had been taken under his direction during the solar eclipses of I 893 and 1896 , and of a series of photographs of stellar spectra which he had commenced at Kensington about I890. Several elements were investigated over a long range of spectrum, and numerous additional lines were found to be intensified on passing from the arc to the spark spectrum, or which only appeared in the spark. .These were designated "enhanced lines," and the work at once led to the definite assignment of origins to many chromospheric and stellar lines which had previously resisted explanation. But this was not all; the enhanced lines were shown to belong to a special class which were only fully developed at high temperatures, so that they gave valuable evidence of physical conditions in the atmospheres of the sun and stars as well as of their chemical constitutions. It would scarcely be too much to claim that this further work on enhanced lines introduced a new principle into astronomical spectroscopy, inasmuch as it justified the chemical identification of celestial spectra which could not be completely reproduced in the laboratory. The only assumption it was necessary to make was that the series of changes indicated in the flame, arc, and spark would be continued if still more powerful means of excitation were available, so that at sufficiently high temperatures the enhanced lines would be the sole survivors. In accordance with his views on dissociation, and for convenience of reference, the enhanced lines were designated "proto-metallic" lines, and attributed to "proto-metals," which were regarded as simplified forms of the vapours which yielded the arc lines.

Apart from any special views as to the cause of their appearance, however, the discovery of enhanced lines has proved to be of the first importance in astrophysical inquiries, and the tables of such lines which were compiled at South Kensington have been much utilised by astronomers throughout the world. Among other applications, as Lockyer was the first to show, the interpretation of the spectra of new stars in their early stages is almost entirely dependent upon a knowledge of the enhanced lines of iron, titanium, and other elements. In collaboration with his assistants, Lockyer showed later that enhanced lines were also developed under the action of strong electrical discharges in non-metallic elements, including silicon, carbon, sulphur, and nitrogen, and the lines observed in these experiments have also led to important celestial identifications. There can be little doubt that the continuation of 
these investigations, as in Fowler's experiments on helium and oxygen, and Merton's further work on carbon, will yield results of high value in the interpretation of the spectra of stars at the highest stages of temperature, and possibly also of the nebulæ.

Another of the chief subjects which attracted Lockyer during a great part of his life was the classification of stellar spectra, and the order of celestial evolution which might be inferred. He was at first mainly dependent upon stellar observations made by others, but he soon saw the necessity for first-hand data, and, following Pickering's remarkable success with the objective prism, he adopted this form of instrument in most of his work at Kensington, and afterwards at Sidmouth. He early adopted the suggestion made by Tait that in nebulæ and comets the luminosity may be due to solids heated by impact, as well as to heated gas generated by the impacts, and about 1887 developed it into his "meteoritic hypothesis." The fundamental idea is that all selfluminous celestial bodies are composed either of swarms of meteorites, or of masses of meteoritic vapour produced by heat, the heat being developed by condensation due to gravity, and the vapour being finally condensed into a solid globe. The classification of stellar spectra which he based upon this theory has undergone modifications in detail, chiefly in the direction of subdivision and more complete definition of the criteria for the various stellar groups; but the essential idea has remained unchanged throughout. In common with other astronomers, Lockyer adopted the view that the spectroscopic differences between the various classes of stars are mainly due to differences of temperature, but, unlike most of them, he insisted that in place of a single line of evolution from hot (white) to cool (red) stars the progression must be from cool to hot stars and back again to cool stars. That is, in accordance with the theory of condensing swarms of meteorites or masses of gas, the classification made a distinction between stars of increasing temperature and those which are on the down-grade towards the extinction of luminosity.

Some of the carlier evidence for the separation of the stars on the two branches of the "temperature curve" which Lockyer pictured may be of doubtful validity, but the valuable photographic data accumulated later, in combination with laboratory researches, placed his classification on a much firmer basis. It was found, for example, that when stars at any given stage of temperature were brought together by reference to the relative intensities of enhanced and arc lines, they were definitely divisible into two groups, showing that the spectra were dependent in part upon physical conditions other than those imposed by temperature alone. This difference was attributed to differences in the state of condensation, one group being less condensed than the other, and therefore to be considered as being in an earlier stage of evolution, notwithstanding equality of temperature. The Harvard classification, which NO. 2652 , VOL. IO5] has been adopted by most astronomers, is along one line of temperature only, and accordingly disregards this difference. It is clearly of great importance, however, that the difference should be taken into account in questions relating to stellar distribution and other matters connected with the structure of the sidereal universe, and it was a source of profound regret to Lockyer that greater attention was not given to it. In the case of the helium stars, however, Lockyer's classification has received substantial corroboration from a discussion by Herassimovitch of their radial velocities and absolute magnitudes, in which the catalogues of Lockyer were utilised. Among the results it was shown that the stars which Lockyer had located on the ascending branch of the temperature curve were brighter than those on the descending branch, and, assuming the average masses to be equal, it would follow that the former were of greater volume and lower density than the latter, in accordance with Lockyer's hypothesis.

The theory of stellar evolution put forward a few years ago by Prof. H. N. Russell resembles that of Lockyer in its main outlines, though based mainly on deductions as to the densities and absolute magnitudes of the stars. The criteria are thus somewhat different in the two cases, but there can be little doubt that in one form or other the recognition of an ascending, as well as of a descending, line of stellar temperatures will take an important place in the astronomy of the future.

The observation of total eclipses of the sun also occupied much of Lockyer's attention. He personally took part in nine eclipse expeditions, and was responsible for several others in which the observations were undertaken by his assistants. On several occasions, when H.M. ships were detailed to assist the expeditions, his exceptional organising ability enabled him effectively to utilise the services of officers and men so as to cover the widest possible range of observations. The outstanding feature of his work in this connection, however, was the introduction and use, first of a visual spectroscope without slit or collimator, and afterwards, when photographic methods could be adopted, of the prismatic camera. With instruments of this type he was able clearly to differentiate between the coronal and chromospheric radiations, and, besides detecting several new coronal lines, he obtained splendid records of the "flash" spectrum. He was thus able to determine the various heights to which the different vapours extended, and he identified a multitude of the bright lines with enhanced lines which he had so diligently investigated in the laboratory.

Lockyer would have been the last to claim that his work was wholly free from errors, but it was almost invariably of a stimulating character, and has played a leading part in the development of the science of astrophysics practically from its very beginning. Much of his work will have an enduring place in the history of the science to which he devoted his great gifts. 